\title{
Bezoar gástrico, una aproximación al manejo
}

\author{
Christian von Mühlenbrock P. ${ }^{3,4}$, Tomás San Martín C. ${ }^{5}$ y Gabriel Mezzano P. ${ }^{1,2}$
}

\section{Gastric bezoar, an approach to medical management}

The presence of bezoars in daily clinical practice is a event that poses a challenge both diagnostic, due to the associated factors in its development, as well as therapeutic. The management of this entity is associated with medical, endoscopic and surgical procedures and it is necessary to know its usefulness in different clinical scenarios. The role of carbonated beverages is increasingly accepted given its low cost, wide availability and high efficiency. For the aforementioned, it seems important to report a series of cases and the management performed.

Key words:

\section{Resumen}

La presencia de bezoares en la práctica clínica diaria es un evento que establece un desafío diagnóstico, por los factores asociados en su desarrollo, como también terapéutico. El manejo de esta patología se asocia a procedimientos médicos, endoscópicos y quirúrgicos siendo necesario conocer su utilidad en distintos escenarios clínicos. El rol de las bebidas carbonatadas es cada vez más aceptado dado su bajo costo, amplia disponibilidad y alta eficacia. Por lo antes señalado, nos parece importante reportar una serie de casos y el manejo realizado.

Palabras clave:
'Departamento de Medicina, Sección Gastroenterología, Hospital del Salvador. Santiago, Chile. ¿Departamento de Medicina, Sección Gastroenterología, Clínica Universidad de los Andes. Santiago, Chile.

Profesor Asistente, Departamento Medicina Interna, Universidad de los Andes. Santiago, Chile.

${ }^{4}$ Residente

Gastroenterología

Hospital Clínico

Universidad de Chile. Santiago, Chile.

Interno, Escuela de

Medicina, Universidad de los Andes. Santiago, Chile.

Recibido:

Aceptado:

Correspondencia a:

Dr. Gabriel Mezzano Puentes

Hospital del Salvador, Av. Salvador 364,

\section{Introducción}

El término bezoar proviene del vocablo árabe badzher que significa antídoto; fueron ellos quienes describieron acumulaciones calculosas en el tubo digestivo tanto en humanos como mamíferos rumiantes. Estos bezoares tendrían propiedades protectoras anti envenenamientos y se usaban como amuletos protectores. Se sabe que la Reina Isabel I de Inglaterra tenía un bezoar que decoraba su corona y Carlos V los usaba ante el miedo de envenenamientos en su corte. En Chile, el historiador Diego Barros Arana, describe que existía un comercio de bezoares extraídos de estómagos de guanacos. Los bezoares eran molidos y mezclados con vino o vinagre en caso de ocurrir una intoxicación, y fueron usados hasta avanzado el

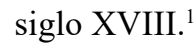

La medicina actual reconoce los bezoares en humanos que consisten en la acumulación de material no digerible en el tracto intestinal, que conforman una masa sólida que impide el paso del bolo alimentario ${ }^{1}$. Su ubicación más frecuente es el estómago, segui- da del intestino delgado. Según su composiciones, se pueden clasificar en 5 diferentes tipos, de este modo, se reconocen los fitobezoares (compuestos por alimentos con base de celulosa), tricobezoares (formados por pelos y restos alimentarios), y menos frecuentemente, están los fármacobezoares, lactobezoares y polibezoares.

Si bien la mayoría de los pacientes son asintomáticos, la acumulación en el tiempo puede producir síntomas asociados a la obstrucción del tracto digestivo que va desde la oclusión parcial a la oclusión total. Las manifestaciones clínicas asociadas a esta patología son: dolor abdominal vago, anorexia, vómitos, baja de peso, masa palpable, obstrucción intestinal, entre otras ${ }^{2}$.

Los factores de riesgo de esta enfermedad son poco claros, pero están descritos aquellos que producen alteración de la anatomía y/o funcionalidad. Así, la presencia de cirugías, tales como cirugías tipo Billroth o bypass gástrico, se asocian a alteraciones anatómicas además de la alteración en la motilidad. Por otro lado, enfermedades sistémicas como diabe-
Providencia, Santiago, Chile. Tel. +56225754000 gmezzano@ clinicauandes.cl 


\section{Caso clínico}

tes, Guillian Barre, enfermedad renal crónica, hipotiroidismo, entre otros pueden favorecer el desarrollo de bezoares por trastornos de motilidad. . $^{3,4}$

El diagnóstico suele realizarse mediante la sospecha clínica y es confirmado por imagenología (desde radiografía de abdomen simple hasta tomografía computarizada), sin embargo, el método de elección es la endoscopia digestiva la cual tiene un rol, en la mayoría de los casos, terapéutico.

El objetivo de presentar este conjunto de casos tiene por fin recordar las distintas alternativas terapéuticas disponibles y el rol terapéutico de las bebidas carbonatadas.

\section{Casos clínicos}

\section{Caso 1}

Hombre de 87 años, con antecedentes de cirugía gástrica tipo Billroth I. Ingresa al servicio de urgencias por episodio de vómitos explosivos de inicio insidioso, asociado a saciedad precoz. Se realizó una radiografía esófago estómago duodeno, que mostró el aspecto de dilatación de la cámara gástrica con la presencia de contenido heterogéneo sin paso de contraste al duodeno. Se complementa estudio con endoscopia digestiva alta que confirmó la presencia de un fitobezoar que compromete la totalidad del remanente gástrico (Figuras 1a). En este contexto, se decidió manejo con Coca-Cola ${ }^{\circledR}$ en infusión de 3 litros en $12 \mathrm{~h}$ por sonda nasogástrica para lograr su di-

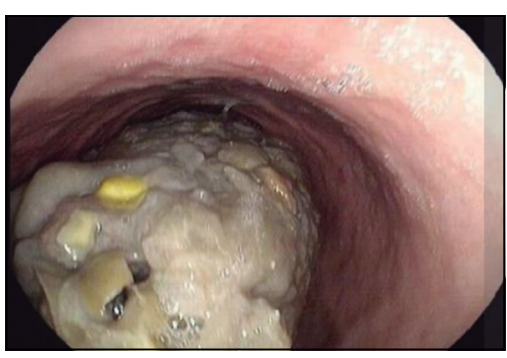

Figura 1a.

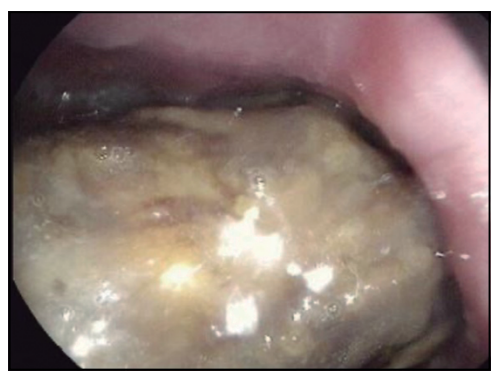

Figura 2a.

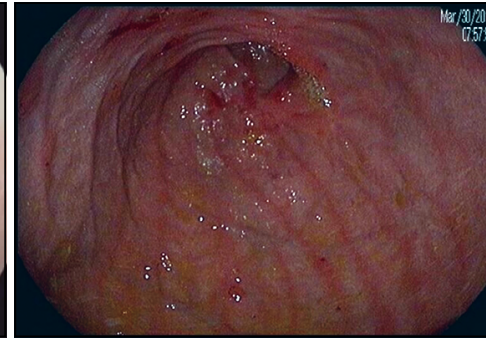

Figura 1 b.

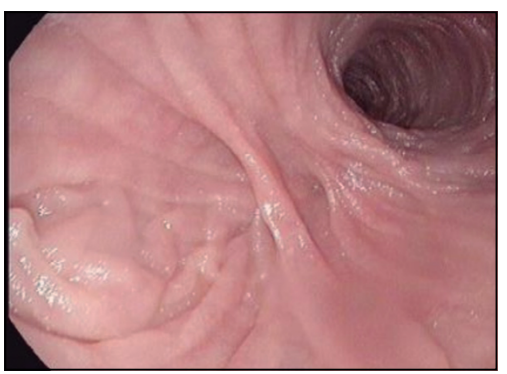

Figura $2 \mathbf{b}$. solución. A las 48 h se logra la mejoría clínica, por lo que se indica alta y control ambulatorio a la semana con una nueva endoscopia (Figura 1b) la cual mostró resolución del cuadro.

\section{Caso 2}

Paciente de género femenino de 41 años, con antecedentes de bypass gástrico realizado en el contexto de obesidad mórbida. Consulta en el servicio de urgencias por dolor epigástrico de 24 h de evolución, de tipo sordo y sin irradiación. El TAC de abdomen y pelvis mostro hallazgos inespecíficos, sin sospecha de la presencia de bezoar gástrico. Ante la persistencia de los síntomas se realizó una endoscopia digestiva alta que mostró la presencia de un fitobezoar gástrico (Figura 2a). En este contexto, se decidió manejo ambulatorio en base de Coca-Cola ${ }^{\circledR}$ administrándose 3 litros vía oral en $12 \mathrm{~h}$ y control endoscópico al 5 to día, el que mostró resolución del bezoar (Figura 2b).

\section{Discusión}

Los bezoares son un hallazgo inhabitual en la práctica clínica diaria, siendo su manejo clínico un desafío ya que se debe definir su causa, composición, riesgo de recurrencia y el mejor tratamiento según la condición del paciente.

El diagnóstico suele realizarse en base a los antecedentes médicos, examen físico, imágenes y endoscopia. En la actualidad, existen distintas terapias usadas que van desde el uso de proquinéticos (metoclopramida), disolución enzimática y extracción por medio de endoscopia o cirugía (en especial para tricobezoares intestinales) ${ }^{5}$.

Uno de los tratamientos más novedosos es la indicación de bebidas carbonatadas, específicamente, Coca-Cola ${ }^{\circledR}$, para su disolución. Descrito por Ladas et $\mathrm{al}^{6,7}$ en el año 2002, incluyeron 46 pacientes con bezoares en los que se logró la disolución del 91,3\% con la administración de Coca-Cola ${ }^{\circledR}$, siendo necesario la resolución quirúrgica en 4 pacientes. El uso de esta bebida carbonatada era más efectivo para fitobezoares que para otros bezoares con tasas de respuesta del $60,6 \%$ versus $23 \%(\mathrm{p}=0,022)$. Otro estudio realizado por Mihai et $\mathrm{al}^{8}$, trató 12 pacientes con fitobezoar con 4.800 cc. de Coca-Cola ${ }^{\circledR}$ observando la disolución completa en el $42 \%(5 / 12)$ y parcial en $42 \%(5 / 12)$. Chung et $\mathrm{al}^{9}$ reportó casos similares con ingesta oral e inyección endoscópica directa con tasa de respuesta similar.

No se conoce claramente el mecanismo de acción ni el volumen óptimo a ser usado. Se plantea que el ácido fosfórico y ácido carbónico de esta bebida, que alcanza un $\mathrm{pH}$ 2,6 permitiría la digestión de fibras; además el bicarbonato de sodio presente tendría un 
efecto mucolítico y las burbujas de $\mathrm{Co}_{2}$ facilitarían la disolución. La terapia combinada química y endoscópica tiene una tasa de éxito mayor al $90 \%$ en bezoares gástricos, dejando relegada la cirugía para casos de fracaso, desarrollo de complicaciones o si la forma de presentación o localización lo hacen inalcanzable al endoscopista. Bezoares distintos a los fitobeazoares, requieren mayor fragmentación endoscópica debido a su mayor consistencia, pese a esto, no se ha encontrado mayor necesidad de cirugía. Faltan estudios para ver efectividad de otras bebidas carbonatadas como Pepsi-Cola ${ }^{\circledR}$ o agua carbonatada que pudiesen tener un efecto lítico similar.

Otros tratamientos descritos en la literatura incluyen el uso de papaína, enzima extraída de la carica papaya, que tendría actividad proteolítica, sin embargo, su efectividad no fue superior al agua en un estudio $^{10}$. Si bien dosis más altas se han reportado como efectivas, se han descrito efectos adversos que incluyen úlceras gástricas y perforación esofágica ${ }^{11}$. Por otro lado, considerando los altos niveles de celulosa presente en fitobezoares, el uso de celulasa (que rompe las uniones glicosídicas), fue descrito en los años setenta como alternativa de tratamiento, aunque es un medicamento difícil de conseguir y estudios han sido discordantes en su efectividad ${ }^{12,13}$. Iwamuro et $\mathrm{al}^{14}$, compararon la efectividad in vitro de Coca-Cola $^{\circledR}$, Coca-Cola ${ }^{\circledR}$ Zero, Celulasa y Papaína, observando que la mayor fragmentación se vio en las muestras expuestas a Coca-Cola ${ }^{\circledR}$, siendo concordante con lo descrito en reportes de casos. Así, con los datos previamente expuestos, se plantea el algoritmo de manejo descrito en Figura 3.

Si bien es una técnica segura, ésta no está exenta de riesgos. Existen reportes de casos en la literatura de obstrucción intestinal secundaria a disolución de bezoares $^{3,15}$. La mayoría de los casos ocurrió con bezoares compuestos de caqui (persimmon bezoar) frecuentes en población asiática, menores a 2-3 centímetros y después de 2 días del inicio de ingesta de la bebida carbonatada. Se cree que el mecanismo sería por el aumento de presión intragástrica impulsando fragmentos de bezoar al píloro e intestino delgado produciendo obstrucción. Dado lo anterior, es que se debe advertir a pacientes de eventuales riesgos y efectuar el procedimiento en un ambiente hospitalizado e

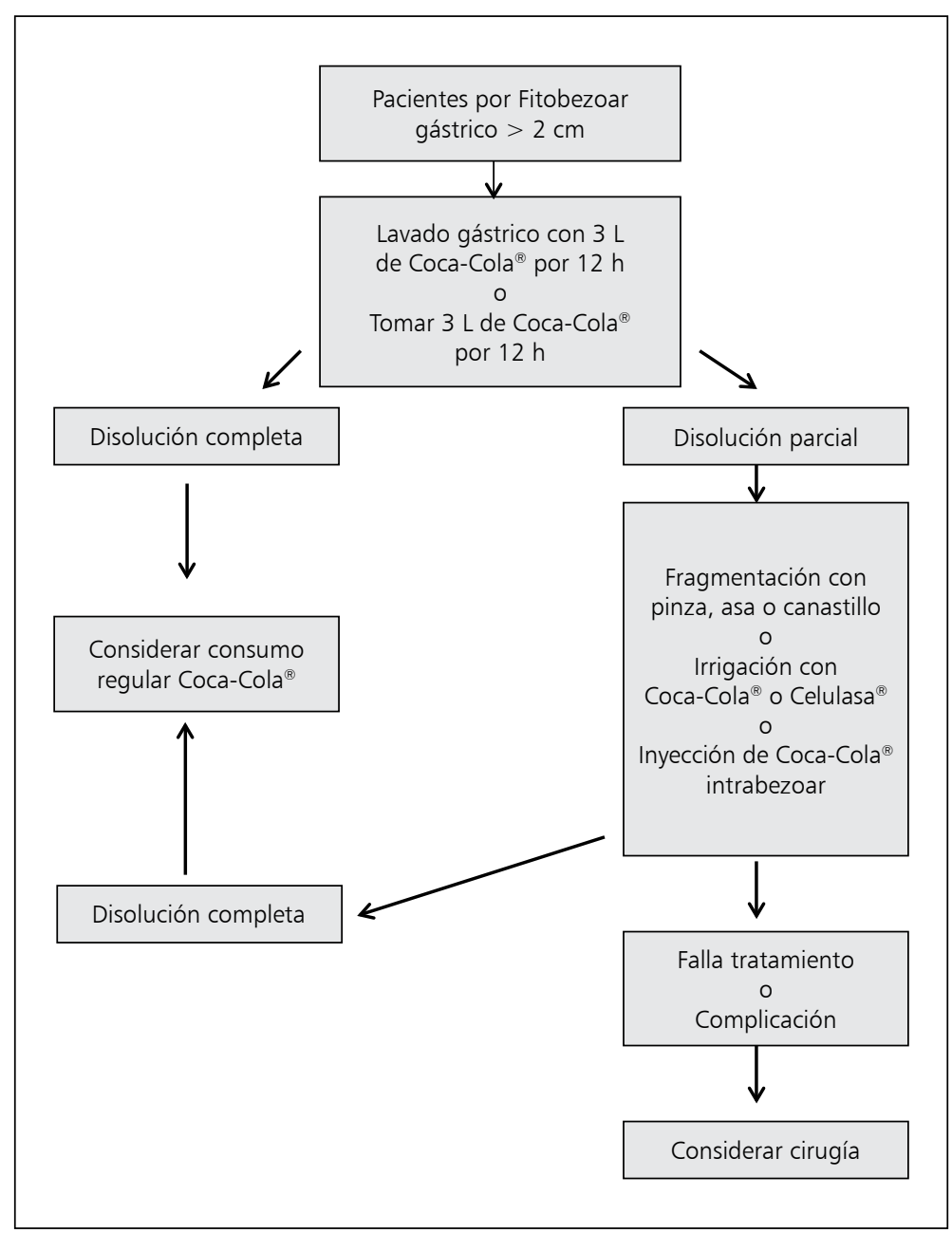

Figura 3. Esquema propuesto de administración de Coca-Cola ${ }^{\circledR}$.

idealmente intentar extracción mecánica en cuerpos extraños menores a 2-3 centímetros ${ }^{16}$.

Por lo expuesto anteriormente, el uso de esta bebida carbonatada, disponible, de fácil administración, segura y de bajo costo, sigue siendo una terapia de elección en casos de bezoares de origen vegetal, ya que aparentemente disminuye la necesidad de intervenciones quirúrgicas. 


\section{Caso clínico}

\section{Referencias}

1.- Espinoza R. Bezoares gastrointestinales: mitos y realidades. Rev Med Chile 2016;144:1073-7.

2.- Iwamuro $M$, Okada $H$, Matsueda $K$, Inaba T, Kusumoto C, Imagawa A. Review of the diagnosis and management of gastrointestinal bezoars. World J Gastrointest Endosc. 2015;7:336-45.

3.- Lu L, Zhang X. Gastric Outlet Obstruction--An Unexpected Complication during Coca-Cola Therapy for a Gastric Bezoar : A Case Report and Literature Review. Intern Med. 2016;55:1085-9.

4.- Caputo F, Barranco R, Bonsignore A, Fraternali Orcioni G, Ventura F. A Rare Case of Fatal Bowel Obstruction Secondary to a Colonic Bezoar. Am J Forensic Med Pathol. 2018;39:38-40.

5.- Iwamuro M, Tanaka S, Shiode J, Imagawa A, Mizuno M. Clinical Characteristics and Treatment Outcomes of Nineteen Japanese Patients with Gastrointestinal Bezoars. Intern Med. 2014;53:1099-105.

6.- Ladas SD, Triantafyllou K, Tzathas C, Tassios P, Rokkas T, Raptis SA. Gastric phytobezoars may be treated by nasogastric Coca-Cola lavage. Eur J Gastroenterol Hepatol. 2002;14:801-3.

7.- Ladas SD, Kamberoglou D, Karamanolis G, Vlachogiannakos J, Zouboulis-va I. Systematic review: Coca-Cola can effectively dissolve gastric phytobezoars as a first-line treatment. Aliment Pharmacol Ther. 2013;37:169-73.

8.- Mihai C, Mihai B, Visele Drug CC. Gastric bezoars-diagnostic and therapeutic challenges. Gastrointestin Liver Dis. 2013;22:109-12.

9.- Chung YW, Han DS, Park YK, Son BK, Paik $\mathrm{CH}$, Jeon $\mathrm{YC}$, et al. Huge gastric diospyrobezoars successfully treated by oral intake and endoscopic injection of Coca-Cola. Dig Liver Dis [Internet]. 2006;38:515-7. Disponible en: https:// doi.org/10.1016/j.dld.2005.10.024

10.- Amit Dwivedi, Fadi Chahin, Sunita Agrawal, Jayesh Patel, Mahmood Khalid YL. Gastric Phytobezoar Treatment Using Meat Tenderizer. Dig Dis Sci. 2001;46:1013-5.

11.- Holsinger Jr. JW, Fuson RL, Sealy WC. Esophageal Perforation Following Meat Impaction and Papain Ingestion. JAMA [Internet]. 1968;204:734-5. Disponible en: https://doi.org/10.1001/ jama.1968.03140210090027.
12.- Gold Jr MH, Patteson III TE, Green GI. Cellulase bezoar injection: a new endoscopic technique. Gastrointest Endosc [Internet]. 1976;22:200-2. Disponible en: https://doi.org/10.1016/ S0016-5107(76)73753-2.

13.- Kramer SJ, Pochapin MB. Gastric Phytobeazoar Dissolution with Ingestion of Diet Coke and Cellulase. Gastroenterol Hepatol (N Y). 2012;8:770-2.

14.- Iwamuro M, Kawai $Y$, Shiraha $H$, Takaki A, Okada H, Yamamoto K. In vitro analysis of gastric phytobezoar dissolubility by coca-cola, cocacola zero, cellulase, and papain. J Clin Gastroenterol. 2014;48:190-1. doi: 10.1097/MCG.0b013e3182a39116.

15.- Ha SS, Lee HS, Jung MK, Jeon SW, Cho CM, Kim SK, Choi YH. Acute Intestinal Obstruction Caused by a Persimmon Phytobezoar after Dissolution Therapy with Coca-Cola FAU Korean J Intern Med. 2007;22(4):300-3. Disponible en: https://doi.org/10.3904/ kjim.2007.22.4.300.

16.- Sharma D, Srivastava M, Babu R, Anand R, Rohtagi A, Thomas S. Laparoscopic treatment of gastric bezoar. JSLS J Soc Laparoendosc Surg. 2010;14:263-7. 